\title{
Médiévales
}

Langues, Textes, Histoire

75 | automne 2018

Traductions du Moyen Âge

\section{« Fatta fedelmente volgarizzare ». Documents traduits et insérés dans la Nuova cronica de Giovanni Villani (Florence, XIV ${ }^{\mathrm{e}}$ siècle)}

"Fatta fedelmente volgarizzare." Translated and inserted documents in Giovanni Villani's Nuova Cronica (Florence, fourteenth century)

Jérémie Rabiot

\section{OpenEdition}

\section{Journals}

Édition électronique

URL : https://journals.openedition.org/medievales/9095

DOI : 10.4000/medievales.9095

ISSN : $1777-5892$

Éditeur

Presses universitaires de Vincennes

Édition imprimée

Date de publication : 15 octobre 2018

Pagination : 49-66

ISBN : 978-2-84292-861-2

ISSN : 0751-2708

\section{Référence électronique}

Jérémie Rabiot, " «Fatta fedelmente volgarizzare ». Documents traduits et insérés dans la Nuova cronica de Giovanni Villani (Florence, xive siècle) », Médiévales [En ligne], 75 | automne 2018, mis en ligne le 15 octobre 2019, consulté le 22 avril 2022. URL : http://journals.openedition.org/medievales/ 9095 ; DOI : https://doi.org/10.4000/medievales.9095 


\section{Jérémie Rabiot}

\section{« Fatta fedelmente volgarizzare " \\ Documents traduits et insérés dans la Nuova cronica de Giovanni Villani (Florence, XIVe siècle)}

Bien que ses activités en aient fait pour un temps une personnalité introduite au sein de l'élite marchande, trois fois prieur dans les années 13101330 et à la tête d'une des compagnies marchandes les plus prospères de cette période, c'est toutefois grâce à sa chronique que Giovanni Villani a vu son nom passer à la postérité. Première grande histoire de Florence en langue vulgaire, la Nuova cronica s'inscrit dans un long siècle d'historiographie florentine : des premiers textes au début du XIII ${ }^{\mathrm{e}}$ siècle, œuvres de clercs ou de juges, rédigées en latin puis traduites, aux premières chroniques originales en langue vulgaire, composées au tournant du nouveau siècle. Lorsqu'il prend la plume, Villani s'appuie donc sur une tradition déjà riche, qu'il ambitionne toutefois de renouveler. Par son ampleur, la Nuova cronica constitue en effet le premier monument de cette historiographie en langue vulgaire : il s'agit, avec la Commedia de Dante qui lui est contemporaine, de l'œuvre vernaculaire la plus imposante jusque-là composée à Florence. Or, choisir le vulgaire, ce n'est pas seulement ériger ce parler au rang de langue de la culture ; c'est aussi choisir de s'adresser à un autre public que celui, lettré, auquel on avait autrefois réservé les chroniques latines. D'emblée, Villani se révèle conscient de la légitimité nouvelle du parler toscan, tout autant que des potentialités ainsi offertes dans la diffusion des savoirs et des idées ${ }^{1}$ : la Nuova cronica n'est pas qu'une œuvre vulgaire, c'est surtout une œuvre de vulgarisation.

Dans son contenu, la chronique suit la trajectoire de son auteur : les derniers livres, rédigés dans les années 1336-1348 et contemporains de la faillite et du déclassement social de Villani, glissent lentement d'une histoire triomphante, celle du Peuple, des Guelfes et des Arts, vers un récit plus

1. Sur le statut de l'italien et son rapport au latin, nous renverrons à A. BARTOLI LANGELI, La scrittura dell'italiano, Bologne, 2000. 
sombre, plus personnel et plus militant. Là, alors que l'histoire a presque rattrapé le présent, l'auteur nourrit son récit d'une diversité de sources nouvelles : lettres de marchands, témoignages oraux, mais aussi documents normatifs ou diplomatiques en latin qu'il fait traduire en vulgaire et recopie. S'il ne s'agit pas d'un fait totalement inédit au Moyen Âge (on pensera à l'importance des chroniques-cartulaires dans l'historiographie monastique des $\mathrm{XI}^{\mathrm{e}}-\mathrm{XII}^{\mathrm{e}}$ siècles ${ }^{2}$ ), intégrer un document à un texte d'histoire constitue toutefois une pratique rare dans l'historiographie communale. Certes, l'importance juridique prise par le document (avec le développement du notariat et la constitution d'archives nécessaires au gouvernement) a eu une influence sur les outils et les méthodes des historiens. Mais, comme le souligne Paolo Cammarosano ${ }^{3}$, historiographie communale et pratique archivistique apparaissent davantage comme deux filons distincts : il ne semble pas y avoir, dit-il, de confluence entre pratique documentaire, constitution d'archives et historiographie communale. Ce qui confère à la Nuova cronica un caractère particulier sur lequel il convient donc de s'interroger.

Partant de l'étude des méthodes et des principes de traduction des sources documentaires retranscrites dans la Nuova cronica, cet article s'intéresse aux stratégies de vulgarisation qui amènent le chroniqueur à s'emparer ainsi de documents en latin pour les traduire et les commenter, participant de ce fait à la diffusion du savoir et des idées. Nous nous attacherons aussi à mettre en lumière les enjeux politiques et sociaux qui sous-tendent cet exercice de communication et montrerons comment celuici s'insère dans un mouvement général de vulgarisation des documents qui marque l'Italie communale dans la première moitié du XIV siècle, à un moment où apparaît une communication publique en langue vulgaire.

\section{Les objectifs de la vulgarisation à travers les " prologues de traduction "}

On dénombre en tout six documents traduits dans la Nuova cronica. Nous noterons au préalable que l'ensemble de ces documents concerne une période assez brève dans la chronologie de rédaction, soit les quinze dernières années de la chronique :

2. Voir par exemple B. GuENÉE, « Documents insérés et documents abrégés dans la Chronique du Religieux de Saint-Denis », Bibliothèque de l'École des chartes, 152/2 (1994), p. 375-428. Plus récent, P. Chastang, Lire, écrire, transcrire. Le travail des rédacteurs de cartulaires en Bas-Languedoc (XI'-XIII siècles), Paris, 2001.

3. Voir à ce propos P. CAMMAROSANO, «I "Libri iurium" e la memoria storica delle città comunali », dans Il senso della storia nella cultura medievale italiana (1100-1350), Pistoia, 1995, p. 309-325. 
- une bulle pontificale de Jean XXII, la Ne super his datée de 1334 (XII, 19) ;

- un traité d'alliance militaire contractée en 1336 entre Florence et Venise contre le seigneur de Vérone, Mastino della Scala (XII, 50);

- deux lettres du roi Robert de Naples : l'une adressée à la Commune en 1333 (XII, 3) et l'autre envoyée en 1342 au seigneur de Florence, le duc d'Athènes Gautier de Brienne (XIII, 4) ;

- deux échanges avec le roi Louis de Hongrie : un discours d'ambassade lu en sa présence en décembre 1347 (XIII, 109) et une lettre du roi à la Commune de février 1348 (XIII, 114).

Trois de ces documents sont introduits par un court paragraphe, quelques lignes à peine, constituant une sorte de prologue qui offre à voir les principes et les enjeux de la traduction. Le premier prologue se rapporte à la lettre envoyée à la Seigneurie florentine par le roi Robert de Naples, au lendemain de la grande crue de l'Arno (XII, 3). Il s'agit d'un long texte rédigé sur le modèle du sermon, dans lequel le roi assure la Commune de son soutien moral $^{4}$ :

Laquelle [épître] nous parait digne d'être mise en note mot pour mot dans notre œuvre, afin d'en laisser mémoire perpétuelle et pour que la clémence et l'amour sincère que le roi portait à notre Commune soient manifestes à nos citoyens successeurs qui viendront et qui liront, et afin que ceux-ci puissent tirer profit et réconfort des bons et saints exemples et admonitions. [...] Et bien qu'en latin, comme il l'envoya, elle fut plus noble, de plus hauts verbes et de meilleur entendement en raison de son beau latin, il nous parut juste de la faire traduire en vulgaire afin qu'elle suivît notre matière vulgaire et fût utile aux laïcs comme aux lettrés.

Le second prologue sert d'introduction à la traduction de la bulle pontificale de Jean XXII, dictée par le souverain pontife in articulo mortis en décembre 1334, au terme d'une longue querelle à propos de la vision béatifique (XII, 19) ${ }^{5}$ :

4. Giovanni Villani, Nuova cronica, XII, 2 (éd. G. Porta, t. III, Parme, 1991) : « la quale [pistola] in questa nostra opera ci pare degna di mettere in nota verbo a verbo a perpetua memoria, acciò che i nostri successori cittadini che verranno e leggeranno quella, sia manifesta la sua clemenza e sincero amore che ' 1 detto re portava al nostro Comune, e di ciò possano [trarre] uttilità di buoni e santi esempli e amunizioni e conforto [...] ; e con tutto che in latino, come la mandòe, fosse più nobile e di più alti verbi e intendimenti per li belli latini di quella, ci parve di farla volgarizzare, acciò che seguisse la nostra materia volgare, e fosse utile a' laici come a li alletterati. »

5. Nuova cronica, XII, 19: « E a ciò che sia manifesto a chi per li tempi leggerà questa cronica, e non possa avere preso errore per quella oppinione, sì metteremo apresso verbo $\mathrm{a}$ verbo la detta dichiarazione fatta fedelmente volgarizzare, come avemo la copia dal nostro fratello che allora era in corte di Roma. » 
Et afin qu'elle soit connue de celui qui par les temps lira cette chronique, et qu'il ne prenne erreur de cette opinion, aussi reporterons-nous mot pour mot ladite déclaration que nous fîmes fidèlement traduire en vulgaire, ainsi que nous en eûmes la copie par notre frère qui était alors à la cour de Rome.

Le troisième extrait accompagne une autre lettre du roi Robert de Naples (XIII, 4), rédigée en 1342 et destinée cette fois-ci au nouveau seigneur de Florence, le duc d'Athènes Gautier de Brienne ${ }^{6}$ :

Et il convient encore de ne pas oublier de mettre en note une brève lettre d'admonition et de grande sentence qui fut retrouvée dans un de ses coffres après son expulsion de Florence - lettre que lui avait envoyée le roi Robert quand il apprit qu'il s'était emparé de la seigneurie de Florence à son insu et sans son conseil, et que nous fîmes traduire du latin en vulgaire afin de suivre notre style, et qui disait [...].

Une remarque préalable ressortant de ces trois extraits concerne l'identité du traducteur : Villani ne traduit pas, mais fait traduire, ce qui nous renseigne sur sa capacité à lire et comprendre le latin. L'essor de la culture populaire dans la Florence du XIV siècle est lié à une alphabétisation avancée, fruit d'un taux de scolarisation record pour l'époque : près de $70 \%$ d'une classe d'âge selon John Najemy ${ }^{7}$. En outre, les marchands ne se contentent pas d'envoyer leurs enfants apprendre à lire dans les «petites écoles » puis se former au calcul dans les « écoles d'abaque » : Robert Black a montré comment, en cet âge préhumaniste, l'apprentissage du latin fait déjà son entrée dans les cursus des fils de marchands ${ }^{8}$. Villani évalue d'ailleurs en 1338 à plus de six cents le nombre d'élèves inscrits dans les quatre « écoles de grammaire et de logique », c'est-à-dire de latin ${ }^{9}$. Mais, alors sexagénaire, l'auteur appartient à une autre génération. Rien ne permet de supposer que lui-même ait suivi un long apprentissage du latin et, quoi qu'il en soit, si ses connaissances en la matière suffisaient sans doute à aborder le vocabulaire simple et stéréotypé des délibérations des conseils, elles ne lui permettaient pas d'entreprendre seul la traduction de textes plus complexes ou abstraits, comme un sermon ou une bulle.

À travers ces trois prologues, il nous est également donné à voir les objectifs de la traduction. Quelques observations s'imposent à ce propos.

6. Nuova cronica, XIII, 3 : «Ancora nonn-è da dimenticare di mettere in nota una brieve lettera d'amunizione di grande sentenzia, che-ssi trovò in uno suo forziere quando fu cacciato di Firenze, la quale gli avea mandata il re Ruberto come seppe ch'egli avea presa la signoria di Firenze sanza sua saputa o consiglio, la quale di latino facemmo recare in volgare per seguire il nostro stile, la quale dicea $[\ldots] »$.

7. J. NAJEMY, A History of Florence, 1200-1575, Maiden, 2006, p. 45.

8. R. BLACK, «École et société à Florence aux $\mathrm{XIV}^{\mathrm{e}}$ et $\mathrm{XV}^{\mathrm{e}}$ siècles. Le témoignage des ricordanze », Annales. Histoire, Sciences Sociales, 4 (2004), p. 827-846.

9. Nuova cronica, XII, 94. 
Tout d'abord, le choix du vulgaire en lieu et place du latin répond à un enjeu stylistique, non pas pour la valeur intrinsèque du vulgaire, mais plutôt pour conserver à sa chronique sa cohérence linguistique. Il s'agit, nous dit Villani, de « suivre notre style » (XIII, 4 et XIII, 109) ou « notre matière vulgaire » (XII, 3). Du reste, il regrette que la traduction entraîne une perte liée à l'abandon du « beau latin », qui fait du document original une œuvre «plus noble » (XII, 3). Cette remarque souligne également le prestige, inégal à ses yeux, des deux langues, le vulgaire ne faisant pas jeu égal avec le latin. Nous sommes loin des positions défendues dans le De Vulgari eloquentia, dont Villani prend pourtant connaissance dans ces mêmes années 1330 et dans lequel Dante proclame la supériorité du vulgaire, plus « naturel», sur le latin, davantage $«$ artificiel ${ }^{10}$.

Un autre point concerne enfin l'objectif annoncé de la traduction, qui doit faciliter la diffusion et la compréhension du document : le choix du vulgaire découle donc également d'un critère d'utilité et d'efficacité. Cet objectif est clairement exprimé dans le prologue de la première lettre du roi Robert (XII, 3), que Villani dit recopier afin qu'elle soit « utile aux laïcs comme aux lettrés », étant attendu que le lecteur en tirera un enseignement utile. Un objectif qui est par ailleurs réitéré à propos de la traduction de la bulle de Jean XXII, laquelle doit servir à « ne pas prendre erreur » de la précédente doctrine du pape (XII, 19). La traduction des sources diplomatiques a donc une fonction médiatrice entre deux niveaux linguistiques (latin et vulgaire) mais aussi culturels (savant et populaire). Or, dans la Nuova cronica, il s'agit là d'un objectif transposable à l'écriture de l'histoire dans son ensemble. Ce lien entre édification morale et civique et vulgarisation linguistique est exposé en effet dès le premier chapitre de la chronique. L'auteur souligne d'emblée la dimension édifiante de l'histoire en se fixant comme objectif de « donner exemple à ceux qui viendront des mutations et des choses passées, de leurs raisons et de leur pourquoi, afin que ceux-ci s'exercent à adopter les vertus et à fuir les vices, et qu'ils supportent l'adversité avec force d'âme, pour le bien et pour l'état de notre république ». Un objectif qui justifie à ses yeux l'emploi du vulgaire, « afin que les laïcs autant que les lettrés puissent en retirer profit et distraction ${ }^{11} »$.

Le choix du vulgaire est donc dicté par le besoin de mettre à disposition des laïcs et des illettrés (comprendre « ceux qui ne maîtrisent pas le latin ») ce qui jusque-là n'était réservé qu'aux clercs et aux savants

10. P. G. Ricci et P. V. Mengaldo, «De vulgari eloquentia », dans Enciclopedia Dantesca, Rome, t. II, 1970, p. 399-415.

11. Nuova cronica, I, 1 : «per dare esemplo a quegli che saranno delle mutazioni e delle cose passate, e le cagioni, e perché ; acciò ch'eglino si esercitino adoperando le virtudi e schifino i vizii, e l'aversitadi sostegnano con forte animo a bene e stato della nostra repubblica. E però io fedelmente narrerò per questo libro in piano volgare, a ciò che li laici siccome gli aletterati ne possano ritrarre frutto e diletto. » 
(comprendre « ceux qui maîtrisent le latin »). Cette profession de foi, qui acquiert dans le prologue une dimension programmatique et qui sera systématiquement réaffirmée dans les prologues de traduction, souligne la fonction exemplaire de l'histoire, dont il est possible de déduire quelques règles générales adaptables en tous temps et en tous lieux. C'est une histoire pragmatique, faite de préconisations et de mises en garde, qui se rapproche d'autres genres littéraires comme le sermon ou le traité de vertu par exemple, lesquels font déjà la part belle au parler vulgaire depuis un siècle environ ${ }^{12}$. La traduction est à son tour porteuse d'un enseignement qui sera civique, moral ou politique. En ce sens, il convient de considérer la vulgarisation comme une composante à part entière de la méthode historique de Giovanni Villani, au même titre que la compilation ou la glose.

\section{Les principes de vulgarisation dans la Nuova cronica : traduction littérale, abrégée, glosée}

Au-delà des objectifs, les trois prologues nous renseignent encore sur les principes de traduction mis en œuvre par l'auteur. Celui-ci annonce ainsi vouloir fournir une traduction fidèle et littérale, consistant à « fai[re] fidèlement traduire » le document (XII, 19) qu'il suivra « mot à mot » (XII, 3 et 19). Si la prétention de fidélité et de littéralité est un topos de la traduction médiévale, qui transparaît souvent à travers les prologues de traducteur (on pensera à Nicole Oresme ${ }^{13}$ ), la comparaison entre la version originale de la bulle Ne super his de 1334 et sa traduction dans la Nuova cronica suffit à montrer que cette prétention n'est pas un vain mot :

12. Sur l'usage du vulgaire dans la prédication, voir par exemple F. MorenzonI, « Les prédicateurs et leurs langues à la fin du Moyen Âge », dans P. von Moos éd., Zwischen Babel und Pfingsten. Sprachdifferenzen und Gesprächsverständigung in der Vormoderne (8.-16. Jahrhundert), Zürich/Berlin, 2008, p. 501-517 ; ou encore C. Delconno, « La lingua dei predicatori. Tra latino e volgare », dans La predicazione dei frati dalla metà del '200 alla fine del '300. Atti del XXII Convegno internazionale (Assisi, 13-15 ottobre 1994), Spolète, 1995, p. 19-46.

13. Voir par exemple E. Barale, « Le "Prologue du translateur" des Éthiques et des Politiques d'Aristote par Nicole Oresme (1370-1374) », Corpus Eve [en ligne] le 18 octobre 2013 : http://eve.revues.org/634 [consulté le 25 juin 2017] ; DOI : 10.4000/eve.634 


\section{Bulle Ne super his ${ }^{a}$}

Ioannis episcopus, servus servorum Dei. Ad perpetuam rei memoriam.

$\mathrm{Ne}$ super his, que de animabus purgatis separatis a corporibus (an citra resumptionem corporum divinam essentiam illa visione, videlicet quam vocat facialem apostolus, videant) tam per nos quam per nonnullos alios in presentia nostra recitando et allegando sacram Scripturam ac originalia dicta Sanctorum vel alias ratiocinando sepius dicta sunt, aliter quam per nos dicta et intellecta fuerint et intelligantur ac dicantur, auribus valeant fidelium inculcari, ecce quod nostram intentionem, quam cum sancta ecclesia catholica circa hec habemus et habuimus, serie presentium ut sequitur declaramus. Fatemur siquidem et credimus, quod anime purgate separate a corporibus sunt in celo, celorum regno et paradiso et cum Christo in consortio angelorum congregate et vident Deum ac divinam essentiam facie ad faciem clare, in quantum status et condicio compatitur anime separate.

$\mathrm{Si}$ vero alia vel aliter circa materiam huiusmodi per nos dicta [predicta seu scripta] fuerint quoquomodo, illa diximus, predicavimus seu scripsimus, recitando dicta sacre Scripture et sanctorum, et conferendo, et non determinando nec etiam tenendo ; et sic et non aliter illa volumus esse dicta, predicata seu scripta. Insuper si qua alia sermocinando, conferendo, dogmatizando, docendo seu alio quovis modo diximus, predicavimus vel scripsimus circa premissa vel alia que fidem concernunt catholicam, sacram Scripturam aut bonos mores, ea in quantum sunt consona fidei catholice, determinationi Ecclesie, sacre Scripture ac bonis moribus, approbamus, alias autem illa habemus et haberi volumus pro non dictis, predicatis et scriptis, et ea revocamus expresse ac ea que de predicta visione, et omnia alia dicta, predicata et scripta nostra de quacunque materia ubicumque et in quocumque loco ac in quocumque statu, quem habemus vel habuerimus hactenus, submittimus determinationi Ecclesie ac successorum Nostrorum. Nulli ergo, etc., nostre declarationis, confessionis, credulitatis, voluntatum, approbationum, reprobationis et submissionis infrangere, etc.

Datum Avinione III non. Decembris, anno decimo nono.
Nuova cronica, XII, 19

Giovanni vescovo, servo di servi di Dio, a perpetua memoria.

Né sopra quelle cose che dell'anime purgate partite da' corpi, se a la resurrezzione de' corpi la divina essenzia con quella visione, la quale l'Apostolo chiama fiaccole, vegghiamo, sì per noi come per molti altri, in nostra presenzia recitando e allegando la sacra Scrittura e li originali detti de' santi, o per altro modo ragionando, spesse volte dette sono altrementi che per noi dette e intese fossono, e intendansi e dicansi, possano nelli orecchi de' fedeli dubbio e oscurità generare ; ecco la nostra intenzione la quale con la santa Chiesia cattolica intorno a queste cose abbiamo, e abbiamo avuto, per lo tenore delle presenti, come seguita : dichiariamo, confessiamo certamente e crediamo che l'anime purgate partite da' corpi sono ne' cieli de' cieli e in paradiso con Cristo, e in compagnia delli angeli raunate, e veggiono Idio e la divina essenzia faccia a faccia chiaramente, in quanto lo stato e la condizione dell'anima partita dal corpo comporta.

Ese altre cose e quale o per altro modo intorno a questa materia per noi dette predicate, overo scritte fossono, per alcuno modo quelle cose abbiamo dette, predicate, overo scritte, recitando e disputando i detti della sacra Scrittura e de' santi, e così vogliamo essere dette, predicate, e scritte. Anche se alcune altre cose sermocinando, disputando, domatriando, amaestrando, overo per alcuno altro modo dicemmo, e predicamo, o scrivemo intorno a le predette cose, overo altre cose che raguardano la fede cattolica, la sacra Scrittura, overo a' buoni costumi, in quanto sono consone a la fede cattolica e a la determinazione de la Chiesa a la sacra Scrittura e a' buoni costumi, la sponiamo ; altrementi per altro modo quelle cose abbiamo avute, e vogliamo per non dette, predicate e scritte, e quelle revochiamo espressamente ; e le predette tutte cose, e qualunque altre predette e scritte per noi di qualunque mai fatti in ogni luogo, $\mathrm{e}$ in qualunque luogo o in qualunque stato, che abbiamo, e abbiamo avuto da quinci adietro, e sommettiamo a la determinazione de la Chiesa e de' nostri successori.

Data a Vignone a dì III di dicembre, anno XVIIII del nostro pontificato.

a. Éditée par H. DENIFLE dans Chartularium Universitatis parisiensis, t. II, s.1, Paris, 1891, p. 440-441. 
Alors que l'approximation caractérise une grande part de l'activité des copistes, traducteurs et autres compilateurs médiévaux, ici domine le souci de précision. Il est vrai que la proximité entre le latin et l'italien dispense d'un travail sur les équivalents lexicaux. Dans la longue séquence synonymique reportée en italique dans le texte, l'essentiel des termes employés en vulgaire se construisent sur le même radical que le terme latin, dont la forme grammaticale est également conservée, au gérondif (sermocinando $>$ sermocinando) ou au passé (scripsimus $>$ scrivemmo) ${ }^{14}$. Certes, le résultat produit peut paraître maladroit et plutôt lourd (ce que l'auteur reconnaît ailleurs), mais, dans l'ensemble, le texte traduit est assez fidèle à l'original - mises à part quelques erreurs grossières comme celle, étonnante quand on sait l'importance de la question dans les débats autour de la vision béatifique ${ }^{15}$, qui transforme la « vision faciale » (« illa visione, videlicet quam vocat facialem apostolus ») en une improbable «vision flambeau » (« quella visione, la quale l'Apostolo chiama fiaccole »)... Une erreur qui ne remet toutefois pas en cause la fidélité globale de la traduction.

Si la littéralité est revendiquée dans ce chapitre, le traducteur peut ailleurs intervenir sur le texte en l'abrégeant ou en l'amplifiant. Le traité d'alliance conclu en 1336 avec Venise constitue ainsi un cas intéressant de traduction tout à la fois fidèle et abrégée ${ }^{16}$ :

MCCCXXXVI, indizione IIII, a dì XXI di giugno, la lega tra 'l Comune di Vinegia e di Firenze fu fatta a Vinegia per li sindachi de' detti Comuni con questi patti.

In prima fecero tra lloro lega, compagnia e unità, la quale duri dal detto dì infino a la festa di san Michele di settembre che viene, e da la detta festa ad uno anno ;

E che per li detti Comuni si soldino $\mathrm{II}^{\mathrm{M}}$ cavalieri e $\mathrm{II}^{\mathrm{M}}$ pedoni al presente, i quali steano a far guerra in trevigiana e veronese ; e quando parrà a' detti Comuni, se ne soldino maggiore quantità ;

E che tutte le mende de' cavalli e ogni spesa che occorresse si debbiano pagare comunemente ;

E che per la detta guerra fare si debbia tenere uno capitano di guerra a comuni spese ;

14. Deux verbes en revanche exigent un effort d'adaptation pour des raisons de clarté : le latin docere ( «enseigner, instruire »), dont le parler vulgaire n'a pas conservé d'équivalent, est remplacé par un amaestrare plus courant, tandis que le latin conferre ( mettre en commun des propos ») est rendu par le vulgaire disputare («s'opposer, défendre son opinion »), sans doute pour éviter la confusion avec le sens pris en vulgaire par le terme conferire ( conférer, attribuer $\gg$ ).

15. Sur la question, nous renverrons à l'ouvrage de C. Trottmann, La Vision béatifique, des disputes scolastiques à sa définition par Benoît XII, Rome, 1995. Sur la réception du début par Villani, voir J. RABIOT, Écrire, comprendre et expliquer l'histoire de son temps au XIV siècle. Étude des livres XI à XIII de la Nuova cronica de Giovanni Villani, Montpellier, 2018, p. 175.

16. Nuova cronica, XII, 50. 
E che per lo Comune di Firenze si mandino uno o due cittadini a stare a Vinegia o dove bisognerà, e abbiano balìa con quelli che-ssi eleggeranno per lo Comune di Vinegia di crescere e menomare i detti soldati come a.lloro parrà, e a potere spendere per fare rubellare le terre che si tengono sotto la segnoria di quelli de la Scala ;

E che sia licito al Comune di Vinegia e di Firenze possano tenere per fare la detta guerra due cittadini e sue bandiere, come a' detti Comuni piacerà ; e abbia il capitano de la guerra pieno arbitrio ; e che per tempo di tre mesi, anzi la fine de la detta lega, si convengano insieme ambasciadori de' detti Comuni a prolungare o non prolungare la lega predetta ;

E che il Comune di Firenze faccia viva guerra a la città di Lucca ; e s'ella s'avesse, facciano guerra a Parma ;

E che i detti Comuni, o alcuno di quelli, non faranno pace, triegua, o terranno alcuno trattato con quelli de la Scala, se non fosse di coscienza e di volontà di ciascuno di detti Comuni.

Une chose remarquable dans ce chapitre est que les formes de l'acte original semblent en partie conservées. La rubrique copie en effet l'introduction du protocole initial, en reprenant la datation selon les formes habituelles des chancelleries (incluant l'indiction). Si le protocole final est ignoré, le contenu de l'acte est reporté de façon relativement fidèle, certes dans une version courte mais respectant visiblement la forme du document puisque chacun des neuf items du texte est énuméré de façon distincte. Si l'on s'en tient à la chronique, le chapitre pourrait donner l'impression de traduire à la lettre le document original. Or une copie de l'acte conservée à l'Archivio di Stato de Florence permet de comparer les deux versions ${ }^{17}$. Les raisons ayant poussé le traducteur à abréger apparaissent au premier coup d'œil : long de deux pages et demie, l'acte est bien trop volumineux pour être traduit in extenso. Le chroniqueur en fournit donc une version fortement résumée. Nous limiterons ici la comparaison à la première clause du deuxième item, qui fixe le montant des troupes fournies par les deux communes :

ASF, Capitoli, XXV, f ${ }^{\circ}$ 2r.

Item, quod predicta communia Venetiarum et Florentie seu per illos qui ad huc per dicta communia fuerint deputati et ad communes expenses ipsorum communium accipiantur et soldiçentur quod velocius fieri poterit equitum duomilia et peditum duomilia, qui equites et pedites ponantur in locis communis Venetie que ipsium commune habeat in partibus Trivisine et [Verone] et ab modo qui guerram faciant et procedant guerriçando per partes Marchie Trivisine et Verone sicut partibus utilius apparebit.

\section{Nuova cronica, XII 51}

E che per li detti Comuni si soldino $\mathrm{II}^{\mathrm{M}}$ cavalieri e II $^{\mathrm{M}}$ pedoni al presente, i quali steano a far guerra in trevigiana e veronese $[\ldots]$.

17. Firenze, Archivio di Stato, Capitoli, 25, f. 51v-53r. 
Dans cet extrait, relativement long dans le texte original, le traducteur évacue les répétitions synonymiques caractéristiques des actes médiévaux (« accipiantur et soldiçentur, qui guerram faciant et procedant guerriçando »), de même que les formules redondantes et de pure forme (« seu per illos qui ad huc per dicta communia fuerint deputati »). L'économie se fait surtout sur la suppression des clauses secondaires, afin de ne retenir que l'information principale. Enfin, le vocabulaire de l'acte original est conservé par le traducteur (voir les italiques), qui en respecte également la syntaxe. Le texte ainsi produit est certes réduit des deux tiers, mais il ne trahit en rien ni le sens ni la forme et demeure finalement assez fidèle au document original.

Dernier cas de figure, enfin, le texte d'origine peut être interprété et amplifié dans sa traduction : c'est le rôle de la glose. C'est peut-être le cas de la seconde lettre du roi Robert, que Villani dit avoir été retrouvée dans un coffre du duc d'Athènes après son expulsion. Dans la version éditée par Giuseppe Porta, le texte présente une série d'explications introduites par le lemme cioè typique de la glose. Le texte est le suivant, les parties en question étant reportées en italique :

Non senno, non vertù, non lunga amistà, non servigi a meritare, non
vendicatogli di loro onte, t'ha fatto signore de' Fiorentini, ma·lla loro grande
discordia e il loro grave stato, di che se' loro più tenuto, considerando
l'amore che t'hanno mostrato, credendosi riposare nelle tue braccia. Il
modo ch'hai a tenere a volerli bene governare si è questo. Che·tti ritenghi
col popolo che prima reggea, e reggiti per lo loro consiglio, non loro per
lo tuo ; fortifica giustizia e i loro ordini, e come per loro si governavano
per sette, fa' che per te si governino per diece, cioè numero comune, che
lega in sé tutti i singulari numeri, ciò vuol dire no·lli reggere per sette né
divisi, ma a comune. Abbiamo inteso che traesti quelli rettori della casa
della loro abitazione, ciò̀ de' priori, nel palagio del popolo fatto per loro
contentamento del propio ; rimettilivi, e abiterai nel palagio ove abitava
nostro figliuolo, cioè nel palagio della podestà, ove abitava il duca di
Calavra, quando fu signore in Firenze. E se questo non farai, non ci pare
che·ttua salute si possa stendere inanzi per ispazio di molto tempo.

Re di Gerusalem e di Cicilia.

Data a Napoli a dì XVIIII di settembre MCCCXLII,... indizione.

Le cas de ce document pose toutefois plusieurs questions. Tout d'abord, une série de manuscrits ignore les gloses en question et propose une version courte de la lettre, ce qui interroge sur leur authenticité ${ }^{18}$. Un second point concerne la langue : si la métaphore numérique (« come per

18. Cf. J. RABIот, Écrire, comprendre et expliquer..., p. 63. 
loro si governavano per sette, fa' che per te si governino per diece ») est rendue possible en vulgaire par l'homophonie entre le mot « secte, faction » (it. setta, pluriel sette) et le chiffre «sept» (it. sette), les équivalents latins (secta et septem) ne rendent pas ce jeu de mots ${ }^{19}$. Enfin, l'espace laissé en blanc à la place du numéro de l'indiction est étonnant : est-il possible que la chancellerie du roi ait envoyé la lettre sans disposer d'une information aussi courante dans les usages diplomatiques ? Ces éléments, ainsi que les circonstances troubles dans lesquelles l'auteur dit avoir mis la main sur le document, laissent entrevoir une autre hypothèse. Il pourrait s'agir ici d'un faux, forgé par le chroniqueur ou par la Commune, destiné à dédouaner la mémoire du roi de Naples de l'expérience malheureuse de la seigneurie de Gautier de Brienne, son ancien officier. Celle-ci apparaîtrait alors comme une parenthèse dans l'histoire institutionnelle de la commune, indépendante de la bonne volonté du roi. Cette hypothèse ferait alors sortir ce texte du champ de la traduction pour le faire entrer dans celui de la propagande.

\section{La vulgarisation, support de stratégies publiques ou privées}

Traduction littérale, traduction abrégée, traduction glosée : les stratégies varient donc en fonction des exemples évoqués. Un principe demeure toutefois constant : la fidélité à la forme et aux marqueurs d'authenticité, qui accentue le caractère documentaire de la traduction ${ }^{20}$. Si celle-ci pourrait au premier abord faire penser à une démarche proto-scientifique, il ne faut toutefois pas surinterpréter la méthode de l'auteur. Les actes traduits et recopiés ne servent pas à proprement parler de sources, si l'on entend par là un document original à partir desquels l'historien construit un récit prétendant à l'objectivité. De fait, le contenu des documents traduits n'apporte généralement rien de factuel. Mais si l'on déplace le regard sur la force sémantique de la correspondance plutôt que sur son contenu, alors ces traductions prennent du sens. C'est donc davantage la publicité conférée au document par sa vulgarisation qui nous intéresse. Un exemple nous permettra d'éclairer quels peuvent être, pour le chroniqueur, les enjeux de la traduction, et de montrer comment celle-ci obéit à des stratégies à la fois publiques et privées, mêlant dans un récit engagé la défense des intérêts politiques de la Commune guelfe à ceux, plus personnels, du marchand en faillite.

19. Si les dictionnaires de latin médiéval évoquent bien une évolution du lemme secta en septa en milieu italien (voir le Glossarium mediae et infimae latinatis de Du CANGE), les occurrences sont rares et la correspondance imparfaite.

20. Cf. par exemple Nuova cronica, XII, 3 : « Ai nobili e savi uomini priori dell'arti, e gonfaloniere di iustizia, consiglio e Comune della città di Firenze, amici diletti e devoti suoi, Ruberto per la grazia di Dio di Ierusalem e di Cecilia re, salute e amore sincero. [...] Data a Napoli sotto il nostro secreto anello, di II di dicembre, seconda indizione, anni MCCCXXXIII. » 
Le chapitre XIII, 109 est la retranscription d'un discours « in gramatica con molti alti latini », autrement dit « en latin et de belle composition », mais là encore que l'on a « fait vulgariser pour suivre le style ». Il fut prononcé par un ambassadeur de la Commune de Florence devant le roi Louis de Hongrie en décembre 1347, dans le contexte des luttes entre les deux branches de la dynastie angevine pour le trône napolitain, après l'assassinat du roi consort André. À ce moment-là, la Commune fait depuis plusieurs semaines la sourde oreille au parti légitimiste conduit par la reine Jeanne et le prince Louis de Tarente, qui lui réclament de l'aide contre le représentant de la branche aînée, Louis de Hongrie. L'Archivio di Stato de Florence a conservé, à partir du 23 septembre 1347, les demandes répétées de la couronne napolitaine d'envois de soldats, conseillers ou argent. Mais à Florence, la Seigneurie refuse de s'engager et préfère faire preuve d'attentisme, décidant même d'aller sonder les intentions de Louis de Hongrie. À la mi-décembre, une ambassade rencontre le roi à Rimini. Or, une lettre retient notre attention : envoyée le $1^{\text {er }}$ octobre par le roi et la reine de Naples, elle constitue une sorte d'abrégé historique de l'alliance florentino-angevine, dans lequel les souverains rappellent les faits d'armes réalisés par leurs aïeux au service du guelfisme toscan ${ }^{21}$. Fait remarquable, le discours lu en latin par le juge Tommaso Corsini devant le roi de Hongrie (angevin lui aussi), puis traduit par Villani, reprend de façon quasi littérale cette lettre écrite deux mois plus tôt par Louis de Tarente - à une époque où aucune archive en ligne ne permettait de relever ce genre de plagiat... Un cas de recyclage de la matière documentaire qui en dit long sur la valeur des archives communales, dans lesquels les communicants peuvent venir librement puiser l'inspiration.

Venons-en maintenant à la traduction que Villani offre de ce discours et interrogeons-nous sur les stratégies qui la motivent. Le choix de traduire ce texte laisse clairement transparaitre le soutien du chroniqueur à l'option hongroise contre ceux qu'il accuse d'avoir précipité le royaume de Naples dans l'anarchie. En un mot, face à la tentation neutraliste de la Seigneurie, Villani soutient l'engagement aux côtés de Louis de Hongrie. Surtout, il souhaite faire connaître son choix. Mais pour lui, l'enjeu n'est pas seulement la préservation d'un pouvoir fort à la tête de l'alliance guelfe comme garantie de la sécurité florentine en Toscane. Il peut s'agir d'abord du rétablissement de l'ordre public en Italie du Sud, condition nécessaire à la poursuite des échanges avec un espace économique majeur pour les compagnies florentines dans lequel, dit-il, « les chemins [sont] livrés aux voleurs, sans ordre ni justice », empêchant ainsi le commerce ${ }^{22}$. Mais on peut aussi évoquer une raison plus personnelle à ce choix : le remplacement de l'État aurait en effet mis hors-jeu une partie de la noblesse napolitaine,

21. Firenze, Archivio di Stato, Capitoli, 16, f. 105r-106r. La demande est réitérée à cinq reprises au moins entre novembre 1347 et février 1348 (f. 107-110).

22. Nuova cronica, XIII, 52. 
profitant ainsi aux marchands florentins, à l'instar de Villani, auprès desquels nombre de ces nobles réclamait le remboursement de créances que la faillite des compagnies avait rendu impossible. Il est en effet significatif de retrouver, parmi les personnes nommément accusées dans la Nuova cronica d'être impliquées dans l'assassinat du roi André, le même nom (Pace da Tropea) que l'on retrouve tout au long de la procédure de mise en faillite de la compagnie de Villani comme membre de la commission nommée pour liquider ses actifs ${ }^{23}$. La diffusion du document n'est donc pas anodine, et certainement motivée par autre chose que sa «belle composition». Sa traduction vient ainsi en soutien d'un discours militant et prolonge les débats en dehors des conseils de la Commune. Bien que marginalisé au sein des élites dirigeantes et mis en difficulté sur le plan judiciaire, Giovanni Villani continue, par l'écrit, de s'investir dans la vie publique de sa Commune.

\section{La traduction comme outil de la communication politique}

Quelles que soient les raisons qui en motivent la diffusion, reste encore à savoir comment Villani fait l'acquisition des documents qu'il fait traduire. À l'instar du traité de 1336, qu'il dit avoir tiré des « actes de la Commune », une partie des documents traduits proviendrait des archives communales. Dépendant alors de la Chambre, celles-ci sont gérées par des « gardiens des actes » qui ont l'obligation de mettre les documents conservés à la disposition du public, sur simple demande et parfois en en fournissant une copie $^{24}$. Pour qui en connaît les parcours, les archives communales sont donc ouvertes. Encore convient-il de distinguer une source d'archives, toujours documentaire, d'une source documentaire, qui n'est pas nécessairement tirée des archives. Or certains de ces documents, avant d'être recueillis par Villani, ont déjà circulé par d'autres voies et dans d'autres circonstances : la bulle de 1334 a été récupérée directement en cour d'Avignon par le frère du chroniqueur, tandis que la lettre du roi Robert de 1342 fut retrouvée dans un coffre au moment du soulèvement de la ville. Dans ces deux cas, et dans d'autres sans doute, Villani n'est qu'un maillon de la chaîne de diffusion du document, sans en être à l'initiative. On aborde alors la question des stratégies liées à la publicité du document, question qui englobe celle de sa traduction.

23. Cf. N. Coulet, Affaires d'argent et affaires de famille en Haute-Provence au XIV siècle. Le dossier du procès de Sybille de Cabris contre Matteo Villani et la compagnie des Buonaccorsi, Rome, 1992, p. 31.

24. Quelques documents, les plus importants, sont toutefois scellés par les sceaux des prieurs et consultables uniquement par eux ou sur leur expresse autorisation. $C f$. L. TANZINI, «Il più antico ordinamento della Camera del Comune di Firenze : le "Provvisioni Canonizzate" del $1289 »$, Annali di Storia di Firenze, 1 (2006), p. 139-179. Voir également R. DavidsoHn, Storia di Firenze, t. IV, Florence, 1973, p. 204-205. 
S'agissant de documents d'intérêt public, liés à des enjeux politiques et produits dans des contextes d'intense débat théologique, de grande émotion populaire ou de vive tension politique, il est probable que ces documents ont bénéficié de la part des autorités d'une publicité, peut-être sous la forme de lectures publiques dans une version traduite. Bien que les exemples conservés soient rares, on sait que les textes les plus importants pour la communauté citoyenne étaient lus en place publique dans une version abrégée et traduite ${ }^{25}$ : il est probable que le traité de 1336 corresponde à ce cas de figure ${ }^{26}$. Les périodes d'agitation de la sphère publique peuvent ainsi bouleverser les usages de la communication officielle : à un moment de trouble, où le pouvoir doit imposer un choix ou légitimer une action, tout devient matière à communiquer le plus largement possible, et donc de préférence en vulgaire. À ce titre, novembre 1333, juin 1336 ou juillet 1343 furent, à Florence, d'intenses moments de communication politique et de vulgarisation.

La traduction en vulgaire de documents latins nous éclaire de ce fait sur un aspect particulier de la culture de l'écrit et du document dans l'Italie du bas Moyen Âge. Après la "révolution documentaire » du notaire au $\mathrm{XIII}^{\mathrm{e}}$ siècle $^{27}$, l'œuvre vulgarisatrice de Giovanni Villani prend place dans ce que l'on pourrait qualifier de « révolution vulgaire » du marchand. Si, dans ce milieu, l'usage du piano volgare est acquis dès le début du XIII ${ }^{\mathrm{e}}$ siècle, d'abord à travers les écrits de la pratique puis les écritures privés ${ }^{28}$, l'accession à la culture lettrée de cette nouvelle bourgeoisie urbaine permet également au vulgaire de s'imposer progressivement dans d'autres domaines (historiographie, sciences, littérature), d'abord sous forme de vulgarisations, puis d'œuvres originales. Dans la pratique officielle toutefois, celle de l'administration et du droit, le latin témoigne d'une certaine longévité du fait de l'importance du notariat en Italie - et ce, y compris à Florence où le vulgaire s'était pourtant très tôt imposé29. Exception faite de quelques cas de statuts précocement vulgarisés, la pratique reste encore rare jusqu'au début du XIV ${ }^{\mathrm{e}}$ siècle $^{30}$. À Florence, d'abord circonscrite aux statuts de confraternités

25. F. Salvestrini et L. TAnZINI, « La lingua della legge. I volgarizzamenti di statuti nell'Italia del Basso Medioevo », dans Comunicare nel Medioevo. La conoscenza e l'uso delle lingue nei secoli XII-XV (atti del convegno di studio, Ascoli Piceno, 28-30 novembre 2013), Rome, 2015, p. 250-301 (p. 293).

26. À la différence des autres documents, Villani ne dit pas l'avoir « fait traduire».

27. Voir, entre autres, A. PetrucCi, La scrittura. Ideologia e rappresentazione, Turin, 1986 ; P. Cammarosano, Italia medievale. Struttura e geografia delle fonti scritte, Rome, 1991. Voir également A. Bartoli Langeli, Notai. Scrivere documenti nell'Italia medievale, Rome, 2006.

28. F. BАмBI, Una nuova lingua per il diritto. Il lessico volgare di Andrea Lancia nelle provvisioni fiorentine del 1355-57, Milan, 2009, p. 5.

29. P. FIORELLI, Intorno alle parole del diritto, Milan, 2008, p. 23 sq.

30. On pensera bien entendu aux statuts siennois, vulgarisés dès 1309. $C f$. L. NERI, «Culture et politique à Sienne au début du XIV siècle : le Statut en langue vulgaire de 1309$1310 »$, Médiévales, 22-23 (1992), p. 207-221. 
religieuses (Santa Maria del Carmine en 1280) ou d'institutions marchandes (Arte degli Oliandoli en 1310-1313, Arte di Calimala en 1334, Mercanzia en 1346), l'œuvre de vulgarisation s'étend ensuite aux statuts communaux à partir du second quart du siècle : ordonnances de justice contre les grands en 1324, statuts de la Parte guelfa en 1335, du capitaine du Peuple et du podestat en 1355-1357. L'accélération remarquable des rythmes de production des vulgarisations officielles dans les années 1325-1350 (c'està-dire de façon contemporaine à la rédaction de la chronique) ne peut que faire penser à une politique de communication concertée, laissant apparaître un contexte de proclamation publique des idéaux du bon gouvernement à travers la traduction des textes constitutifs de l'identité communale ${ }^{31}$.

Les efforts de Villani visant à produire des documents officiels en langue vulgaire doivent donc être replacés dans ce double contexte : d'une part, celui de plus grande accessibilité à l'écrit de la part des laïcs non formés au latin (à l'inverse des notaires et juges du XIII ${ }^{\mathrm{e}}$ siècle) ; d'autre part, celui d'une vulgarisation de la culture dans toutes ses dimensions, et notamment politique et civique. Certes, les documents traduits à l'initiative de Villani ne sont pas ceux que la Commune fait vulgariser au même moment : point de grand texte normatif, de statut ou d'ordonnance, mais, pour l'essentiel, des échanges épistolaires entre chancelleries - autrement dit des documents qui concernent moins l'identité politique interne de la Commune populaire des Arts que ses relations extérieures au sein de l'Italie guelfe. Toutefois, cette sensibilité nouvelle du chroniqueur envers le document prend un sens particulier dans ce contexte général d'intensification de la communication politique en vulgaire. Peut-être du reste les traducteurs mis à contribution par Villani furent-ils les mêmes que ceux commissionnés par la Commune : on pensera bien sûr à Andrea Lancia, vulgarisateur des Statuts de 13551357, mais aussi ami proche de Villani et auteur de la traduction de l'Énéide utilisée par ce dernier ${ }^{32}$.

Or, cette appétence pour la vulgarisation ne nous apparaît pas seulement comme un fait linguistique et culturel, mais semble témoigner d'une évolution sociologique plus large. Dans le contexte communal italien, la vulgarisation du document administratif ou diplomatique à des fins publicitaires témoigne de ce que celui-ci n'est plus considéré comme l'affaire des seuls experts du droit, juges et notaires, mais de tout le peuple des laïcs illettrés à qui l'art de la politique s'est ouvert. Il devient donc au contraire un objet d'intérêt public, dont tout le monde doit pouvoir

31. Le parallèle avec Sienne, où les Statuts ont été vulgarisés dès 1309-1310, est intéressant : voir à ce propos F. SALVESTRINI et L. TANZINI, « La lingua della legge... », p. 253258.

32. M. Cerroni, «Lancia, Andrea », dans Dizionario Biografico degli Italiani, 63, 2004 [en ligne] : http://www.treccani.it/enciclopedia/andrea-lancia_(Dizionario-Biografico)/ [consulté le 4 juin 2018]. 
s'emparer pour le discuter ou le contester ${ }^{33}$. De ce fait, on « démocratise » le document, pourrions-nous dire. Et c'est dans ce sens-là, nous semble-t-il, qu'il faut comprendre les prologues de traduction, dans lesquels l'auteur présente la vulgarisation comme un exercice de médiation au service des laïcs dans la cité. Dans la veine des traités de vertu, l'enjeu est certes d'abord individuel (le récit doit être « utile » au lecteur, qui en tirera «profit » sur le plan moral), mais il est également collectif et politique, dans la mesure où cette œuvre édificatrice poursuit comme fin ultime « le bien et l'état de notre république ». C'est cette dimension à la fois publique et publicitaire de la traduction qui nous semble l'élément le plus important de ces exercices de vulgarisation. La Nuova cronica lève ainsi le voile sur l'existence, à la fin du Moyen Âge, d'une « opinion publique » au sens où l'entendait Habermas, lequel situait pourtant la naissance de l'öffentliche Meinung au sein de la bourgeoisie éclairée du XVIII ${ }^{\mathrm{e}}$ siècle, en réaction à l'absolutisme ${ }^{34}$. Car, en réceptionnant, en traduisant et surtout en commentant le document, Villani témoigne bien de la capacité du public médiéval à recevoir la publicité officielle (la propagande), à se positionner en fonction d'elle et à y réagir de façon publique.

Ce programme de médiation politique et culturelle, indépendant de la communication descendante du gouvernement, connut du reste un certain succès si l'on considère la centaine de manuscrits de la Nuova cronica encore conservés aujourd'hui, presque tous de facture florentine et datant pour l'essentiel de la fin du XIV ${ }^{\mathrm{e}}$ siècle $^{35}$. C'est lors de la génération suivante, celle des années 1350-1380, que l'effort de vulgarisation mis en œuvre par Villani aura sans doute eu le plus d'effet. Sans surprise du reste, étant donnée la façon dont les enjeux qui avaient marqué son temps - redéfinition des équilibres politiques à l'échelle de la péninsule ou instabilités sociale et constitutionnelle au sein de la commune - trouvaient écho dans la guerre des Huit Saints (1375-1378), la révolte des Ciompi (1378) ou la restauration oligarchique de $1381^{36}$. En outre, la difficulté à établir un profil type du lecteur de la Nuova cronica à partir des indices de possession des

33. $C f$. F. SAlvestrini et L. TAnZini, « La lingua della legge... », p. 272. Les auteurs remarquent que, dans certaines communes, la vulgarisation des statuts a été perçue comme une alternative au langage des juges et notaires, une marque de méfiance vis-à-vis de ceux qui détenaient le monopole du droit (ce qui ne semble pas être le cas à Florence, où pareil antagonisme ne se retrouve pas).

34. J. Habermas, L'Espace public. Archéologie de la publicité comme dimension constitutive de la société bourgeoise, Paris, 1978. Pour un aperçu critique de cette notion, cf. M. Lits, «L'espace public : concept fondateur de la communication », Hermès, 70 (2014), p. 77-81. Sur l'application du concept habermasien au Moyen Âge, nous renverrons à N. OfFenstadt et P. Boucheron, L'Espace public au Moyen Âge. Débats autour de Jürgen Habermas, Paris, 2015.

35. J. RAвіот, Écrire, comprendre et expliquer..., p. 31-33.

36. Voir J. NAJEMY, A History of Florence..., p. 156-187. 
manuscrits, où les riches patriciens côtoient les petits artisans, montre que, dans les faits, ce public s'étendait bien au-delà de la bourgeoisie marchande préhumaniste, bien au-delà même des cercles savants ou lettrés, mais potentiellement à l'ensemble de la communauté des citoyens détenteurs de droits politiques et, supposément, d'une opinion ${ }^{37}$. Or, bien que l'auteur affirme à plusieurs reprises adresser son œuvre à « ceux qui viendront », la traduction et la retranscription des documents semblent moins obéir, dans la Nuova cronica, à des enjeux de conservation des actes, dans un esprit archivistique destiné à la postérité, qu'à la formation politique des lecteurs par la promotion d'une culture documentaire visant à retranscrire, dans l'espace public et en vulgaire, la pratique diplomatique et normative de la Commune. Nous n'irons certes pas jusqu'à comparer les derniers livres de la chronique, dans lesquels se concentrent les documents traduits et où s'impose un ton de plus en plus polémique, à la presse d'opinion de l'époque moderne : ce qui manque encore, c'est l'instantanéité de la communication, à une époque où les potentialités de diffusion des textes n'ont pas encore été démultipliées par l'imprimerie. Mais la pratique documentaire de Villani nous semble bel et bien avoir comme objectif d'insérer le document dans une sphère publique, c'est-à-dire un espace public virtuel situé hors des lieux publics du gouvernement ou de la communication officielle en latin : autrement dit, dans ce que l'on appellerait le débat public.

Jérémie Rabiot - Gymnase de Münchenstein (Bâle, Suisse), UMR 5648 (CIHAM)

\section{" Fatta fedelmente volgarizzare ". Documents traduits et insérés dans la Nuova cronica de Giovanni Villani (Florence, XIV ${ }^{e}$ siècle)}

Cet article étudie la traduction en vulgaire et la retranscription de documents latins dans la Nuova cronica, une histoire du monde et de la ville de Florence rédigée au XIV ${ }^{e}$ siècle par Giovanni Villani, chroniqueur-marchand. Partant de l'étude des objectifs et des principes de traduction mis en œuvre, il souligne les enjeux politiques et sociaux qui amènent l'auteur à rechercher, traduire et recopier des documents officiels (bulle pontificale, échanges de chancellerie ou traité d'alliance). II s'efforce ainsi de rapprocher ces caractéristiques de l'essor des vulgarisations de statuts communaux, dans un contexte d'intensification de la communication politique en vulgaire dans les cités communales d'Italie.

Florence, Giovanni Villani, Italie, Nuova cronica, traduction, vulgaire

\section{"Fatta fedelmente volgarizzare." Translated and inserted documents in Giovanni Villani's Nuova Cronica (Florence, fourteenth century)}

This paper focuses on the translation into vernacular and the transcription of Latin documents in the Nuova Cronica, a history of the world and of the city of Florence written in the fourteenth century by the chronicler

37. Ibid.,p. 33-35. 
and merchant Giovanni Villani. It studies the objectives and principles of translation adopted here, and highlights the political and social issues that led the author to receive, translate, and copy official documents (papal bull, exchanges of Chancery, or alliance treaty). It therefore aims to link these facts with the increase of vulgarizations of communal statutes, at a time when political communication in vernacular was becoming more widespread in the communes of Italy.

Florence, Italy, Giovanni Villani, Nuova Cronica, translation, vernacular 